sciendo

DOI: 10.2478/BIPMF-2021-0001
BULETINUL INSTITUTULUI POLITEHNIC DIN IAŞI

Publicat de

Universitatea Tehnică „Gheorghe Asachi” din Iaşi Volumul 67 (71), Numărul 1, 2021

Secţia

MATEMATICĂ. MECANICĂ TEORETICĂ. FIZICĂ

\title{
A NEW THEORETICAL MODEL FOR EVALUATING ATRIAL FIBRILLATIONS
}

\section{TEODOR-MARIAN IONESCU ${ }^{1}$, VLAD GHIZDOVĂȚ $T^{2, *}$, IRENA-CRISTINA GRIEROSU $^{2}$, DECEBAL VASINCU ${ }^{3}$ and CIPRIANA ŞTEFĂNESCU ${ }^{2}$}

"Grigore T. Popa" University of Medicine and Pharmacy, Faculty of Medicine, Iaşi, Romania

2"Grigore T. Popa" University of Medicine and Pharmacy, Faculty of Medicine, Biophysics and Medical Physics Department, Iaşi, Romania

3"Grigore T. Popa" University of Medicine and Pharmacy, Faculty of Dental Medicine, Biophysics Department, Iaşi, Romania

Received: February 4, 2021

Accepted for publication: March 29, 2021

Abstract. By using mathematical formulations specific to nonlinear dynamics, we devise an original method for the evaluation of atrial fibrillations. Our results can be used for developing new medical diagnosis procedures and new ways of tracking heart diseases, based on specific patterns.

Key words: ECG; atrial fibrillation; nonlinear dynamics.

\section{Introduction}

Almost 60 years ago, Noble developed a first cardiac cell model based on modified Hodgkin-Huxley equations describing the long-lasting action and pace-maker potential of the Purkinje fibers of the heart (Noble, 1960). Since then, several nonlinear models of electrophysiological dynamics were developed with increasing complexity and specificity (Nayak et al., 2018;

${ }^{*}$ Corresponding author; e-mail: vlad.ghizdovat@umfiasi.ro

This is an Open access article licensed under the Creative Commons Attribution-Non Commercial

- No Dericatives 4.0 International License (CC BY-NV-ND 4.0) 
Vagos et al., 2018)). These models suggested the use of nonlinear method to investigate the electrical activity of the heart. In their pioneering works, Guevara et al. (Guevara et al., 1981) and Ritzenberg et al. (Ritzenberg et al., 1984) introduced nonlinear approaches to heart rhythm analysis and provided evidence of nonlinear behavior in the electrocardiogram. Methods borrowed from the theory of chaos control were successfully applied for the termination of the repolarization alternans to prevent alternans-induced ventricular tachycardia of fibrillation (Christini et al., 2001) and control of electrical turbulence in the heart (Luther et al., 2011).

In a normal heart, the electrical phenomenon originated from the cardiac activity triggers the mechanical phenomenon with a 0.02 second delay. Tracking and recording these phenomena may provide important information regarding the genesis, propagation and development of cardiac activity (Haulica, 2007). Electrocardiography records variations in the electrical potential in the myocardium during a cardiac cycle and represents them as graphic deflections or electrocardiogram waves. Shifts in the activity of different regions are not made simultaneously, but in a predetermined order. Each wave, segment and complex registered by the device is equivalent to the depolarization and repolarization of a certain area or chamber of the heart ( $\mathrm{P}$ waves - atrial depolarization; PQ segment - conduction of the depolarization stimulus; QRS complex represent ventricle depolarization, ST segment - ventricle repolarization; T waves - ventricle repolarization) (Badescu et al., 2016).

A normal ECG is represented by a complex of $\mathrm{P}$ waves, followed by QRS complex and $\mathrm{T}$ wave respecting certain parameters of height, depth, with, rhythm and heart rate.

In case of arrhythmias, the normal rhythm of the myocardium is affected (frequency, regularity or both). Electrophysiologically, rhythm disorders such as fibrillation (characterized by numerous ectopic centers that discharge impulses at a very high rate) would result in an abnormal regional depolarization (atrial and ventricle fibrillation) therefore interfering with the normal cardiac activity. Depending on the fibrillation type the main diagnostic criteria on a 12 lead ECG may vary (atrial fibrillation: absent P waves associated with small "f" fibrillation waves, irregular with variable amplitude and a very high frequency, unequal QRS complexes but with normal morphology; ventricle fibrillation: absence of $\mathrm{P}$ and $\mathrm{T}$ waves and replacement of the QRS complex with fast electrical waves that present morphological, amplitude and duration modification that vary during recording) (Badescu et al., 2016).

The nonlinear dynamics investigation of the electrocardiogram time series can offer important information also for other physiological processes than those specific to cardiology. Thus, experimental results revealed that the heartbeat rate signal present a low-dimensional chaotic state during an epileptic seizure, while before and after the seizure event it presents a complex, aperiodic 
behavior (Su et al., 2008). Also, Young and Benton associated heart rate complexity with ratings of mood, focused attention reaction times, inhibition, and decision time, concluding that nonlinear rather than linear methods of summarizing the heart rate times series offers new perspective in the relation between brain functions and its behavior (Young and Benton, 2015).

In this paper we devise a new method for the evaluation of atrial fibrillations, by using mathematical procedures from nonlinear dynamics.

\section{Methods}

We analyzed ECGs obtained from the open acces PhysioNet database (physionet.org). This is a free collection of physiological signals (PhysioBank), recorded from a wide range of patients, as well as specialized software for viewing and analyzing them. Thus, this database aims to stimulate current research in analyzing complex (often nonlinear) biomedical and physiological signals.

Nonlinear dynamics proposes a vast array of methods for signal analysis. Selecting one is done taking into account the signal specifics and the information one wants to obtain. We will mention only the methods employed in this work.

The Lyapunov exponents associated to a phase space trajectory of a dynamic system are a measure of the average expansion or contraction rate of trajectories around it, i.e., trajectories that arise from very close initial conditions (Arce, 2004). These exponents are asymptotic quantities, locally defined as follows. Let us consider $x(t)$ the trajectory in the phase space of a dynamic system, which starts from an initial condition very close to $x_{0}$ : $x^{\prime}(t=0)=x_{0}^{\prime}$. We define the distance between the two trajectories as $y(t)=\left|x(t)-x^{\prime}(t)\right|$. Considering that this distance varies exponentially with time (this hypothesis must be verified for each case in particular):

$$
y(t)=y(0) e^{\lambda t}
$$

The exponent which appears in this relation, $\lambda$, is called the Lyapunov exponent. If the phase space is $n$-dimensional (there are $n$ independent quantities describing the state of the system), then we can define a Lyapunov exponent for each dimension, therefore $n$ Lyapunov coefficients exist, which, together, form the Lyapunov spectrum (Nayfeh and Balachandran, 2004). If the Lyapunov exponent is positive, it means that there is a high divergence of initial trajectories from neighbouring initial conditions, this being characteristic to chaotic dynamic systems. We usually don't need to calculate all the Lyapunov exponents, because there is a fast algorithm for calculating the highest 
Lyapunov exponent. If this is positive, the system is chaotic; if it is negative, it means that all the other Lyapunov exponents are negative, therefore the system is not chaotic.

The phase space for a system dynamic is usually higher than 3 , so that its graphical representation is impossible. This type of spaces can only be visualized by using projection on subspaces of 3 dimension. Eckman, Kamphorst, and Ruelle developed a method for visualizing the recurrence of state $x_{i}$, in a phase space (Eckmann et al., 1987). Their method allows investigating the system's trajectory in a $m$-dimensional space by representing its two-dimensional recurrences. The recurrence of a state corresponding to the moment $t_{i}$ at a different time $t_{j}$ can be graphically presented by a twodimensional square matrix, in which the dark coloured points represent the recurrence. This is called a recurrence graphic, and it can be defined by the following relation:

$$
R(i, j)=\Theta(\varepsilon-\|\vec{r}(i)-\vec{r}(j)\|), \quad \vec{r}(i) \in R^{m}, \quad i, j=1, \ldots, N
$$

where $N$ is the number of $x_{i}$ states considered, $\varepsilon$ is a threshold distance, and $\Theta$ is the Heaviside step function (Arce, 2004). The recurrence graphic shows both large scale (topology) and reduced scale (texture) characteristics. Topology offers a global impression, which can be characterized as homogenous, period, drift, or disrupted.

In Fig. 1 recurrence maps are drawn, corresponding to "white" noise (random signal), harmonic oscillations (perfect sinusoidal), Brownian motion, and to a healthy patient's ECG (Perc, 2005).

For the quantitative estimation of the structural degree of the recurrence map, the spatial-temporal entropy is calculated, measuring both the spatial and temporal structural degrees. It compares the global distribution of colours along the whole recurrence map with the distribution of colours along each diagonal of the map (Aflori and Dimitriu, 2006). The result is normed and presented in the form of a maximum value percentage, corresponding to a random signal. A $100 \%$ spatial-temporal entropy indicates the absence of any structure (uniform colour distribution), while a value of $0 \%$ for the same entropy means a perfect structuralizing (distinct model in the map, total predictability) (Aflori and Dimitriu, 2006).

In most experimental situations it is very hard to establish the independent coordinates that describe a systems' dynamics, necessary for building the phase space. For this reason, several alternative methods for constructing such a space have been developed, the most well-known one being the delay time method. 


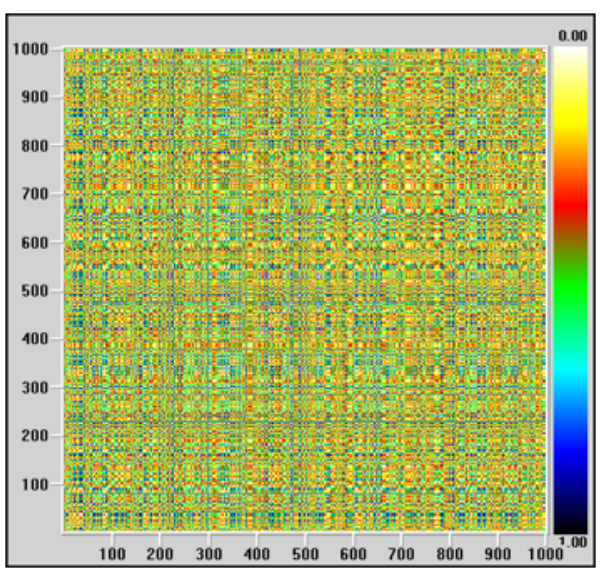

(a)

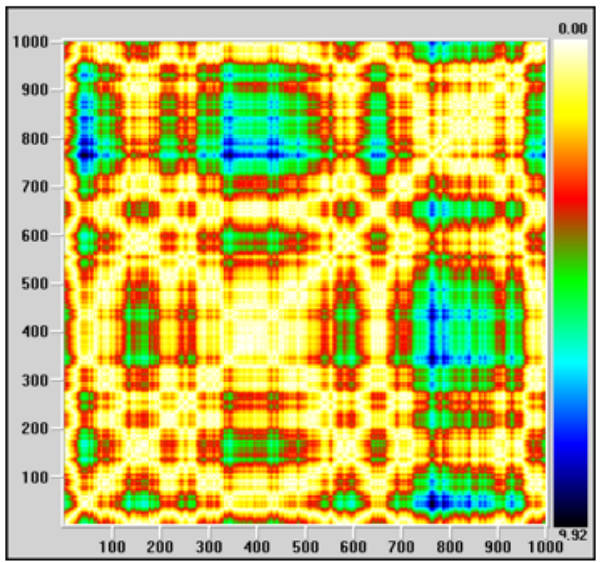

(c)

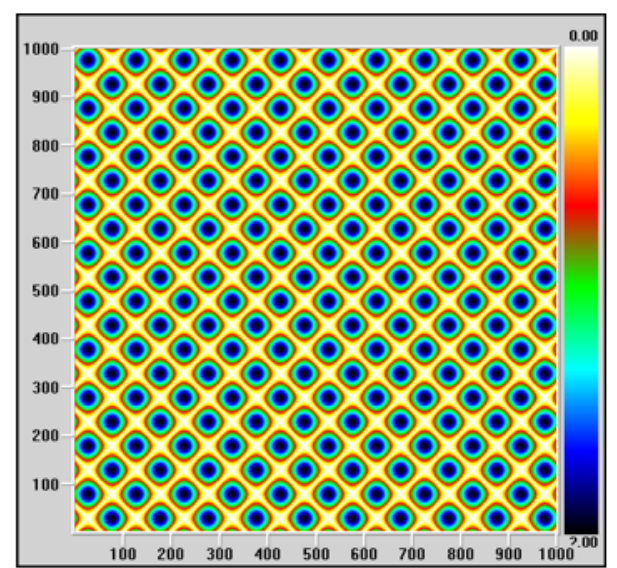

(b)

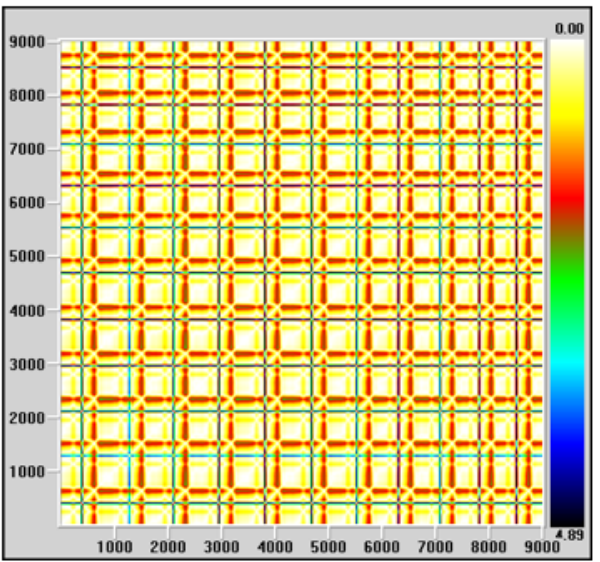

(d)

Fig. 1 - Recurrence maps corresponding to white noise (a), harmonic oscillations $(b)$, Brownian motion $(c)$, and a healthy patient's ECG $(d)$.

Experimentally, most often a single system variable is measured:

$$
s_{n}=s(t)=s\left(t_{0}+n \tau_{s}\right)
$$

where $\tau_{s}$ is the sampling time of the instrument used for measuring the $s$ variable. A series of paper written at the end of the $20^{\text {th }}$ century suggested that the measurement of a single signal could be sufficient to "reconstruct" a phase space in which the system's dynamics is equivalent with the one from the original phase space. Packard et al. demonstrated this equivalency through 
numerical simulations (Packard et al., 1980), while Takens demonstrated it with mathematical rigor (Takens, 1981). As a consequence of this equivalence, the attractor from the reconstructed phase space has the same invariants (like the Lyapunov exponents) as the original one.

In order to reconstruct the independent coordinates of the phase space, Packard and co. proposed using the signal's derivatives (Packard et al., 1980). In this way, the derivates can be approximated with the finite differences:

$$
\begin{gathered}
\frac{d s}{d t}\left(t_{0}+n \tau_{s}\right) \cong \frac{s\left(t_{0}+n \tau_{s}\right)-s\left[t_{0}+(n-1) \tau_{s}\right]}{\tau_{s}} \\
\frac{d^{2} s}{d t^{2}}\left(t_{0}+n \tau_{s}\right) \cong \frac{s\left[t_{0}+(n+1) \tau_{s}\right]-2 s\left(t_{0}+n \tau_{s}\right)}{\tau_{s}^{2}}+\frac{s\left[t_{0}+(n-1) \tau_{s}\right]}{\tau_{s}^{2}}
\end{gathered}
$$

From the above-written formulas it can be observed that, at each new differentiation, new information already contained in the measured signal is added, but at time moments delayed with a multiple of the delay time. This observation lead Packard and co. (Packard et al., 1980), Takens (Takens, 1981), and Ruelle (Ruelle, 1989) to the conclusion that there is no need for derivatives in order to compose a coordinates system that describes the structure of orbits from the phase space. Instead, one can directly use the $s(t+n \tau)$ variables, where $n=1,2, \ldots, d$, and $\tau=k \tau_{s}$ is an appropriately chosen delay time. We can define, thus, the so-called delayed coordinates vectors as:

$$
y_{n}=\left\{s\left(t_{0}+n \tau_{s}\right), s\left(t_{0}+n \tau_{s}+k \tau_{s}\right), \ldots, s\left[t_{0}+n \tau_{s}+k(d-1) \tau_{s}\right]\right\}^{T}
$$

or

$$
y_{n}=\left\{s_{n}, s_{n+k}, s_{n+2 k}, \ldots, s_{n+(d-1) k}\right\}^{T}
$$

The space constructed with the $y_{n}$ vectors is called the reconstructed phase space. According to Takens (Takens, 1981) and Mané (Mané, 1981), the geometrical structure for the dynamics of the systems for which the variable $s$ has been measured can be observed in the reconstructed $d$-dimensional Euclidian space, if $d \geq 2 d_{a}+1$, where $d_{a}$ is the attractor's dimension. In 1991, Sauer et al. had shown that it is sufficient for $d \geq 2 d_{a}$. The parameter $\tau$ is called the delay time, and the integer $d$ is called the immersion dimension.

The immersion dimension must be large enough so that no intersection of orbits reconstructed with themselves (false intersections) should appear. There are several ways for determining this dimension, the most often used one 
being the systems' invariants saturations and the false nearest neighbours method (Nayfeh and Balachandran, 2004).

In theory, the delay time can be arbitrary chosen if we have an infinity of data, not altered by noise. As this is improbable in practice, the value of the delay time must be chosen with care. If this value is too low, the trajectories in the reconstructed phase space will clutter towards the main diagonal, due to the fact that the delayed coordinates are excessively correlated. On the other hand, if the chosen value is too high, an artificial decorrelation can appear, and the delayed coordinates become uncorrelated. The most often used methods for determining the delay time are the autocorrelation function, the average mutual information, and the generalized correlations integral methods (Nayfeh and Balachandran, 2004).

\section{Results and Discussions}

In Fig. 2 the graphical representations of ECG fragments (with a $5 \mathrm{~s}$ duration) corresponding to pre-crisis, first atrial fibrillation (AFIB 1), atrial flutter (AFL), second atrial fibrillation (AFIB 2) and post-crisis, respectively, are presented for the analyzed ECG presented in Methods.

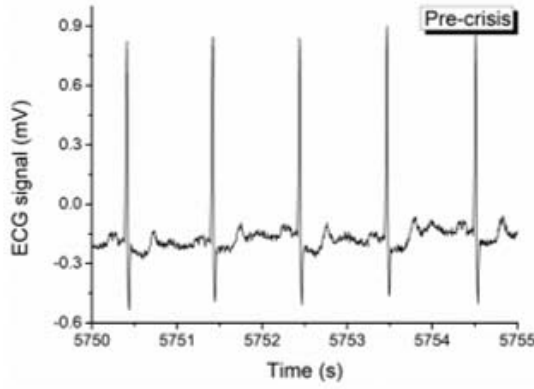

(a)

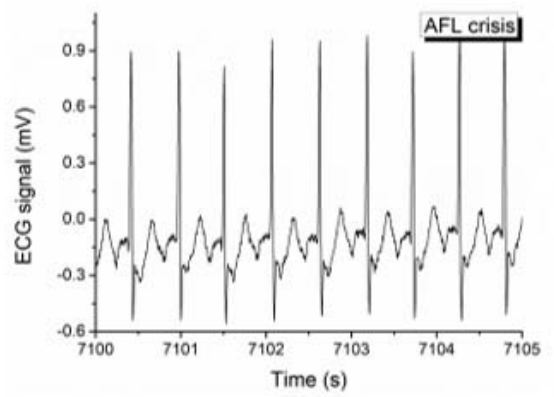

(c)

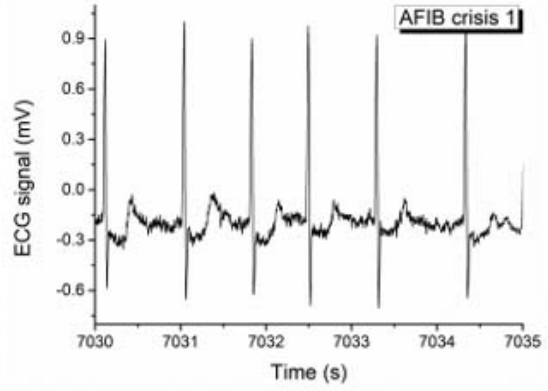

(b)

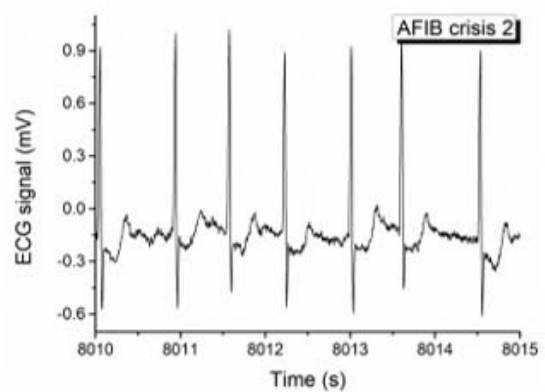

(d) 


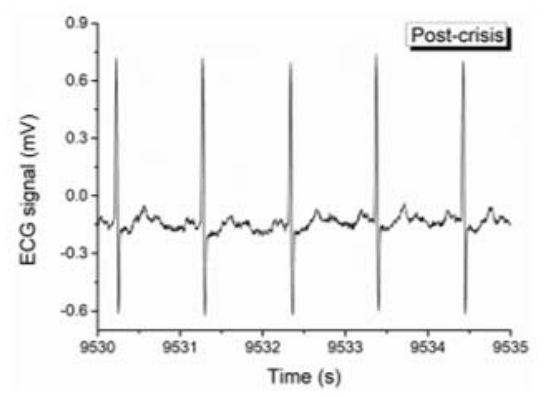

(e)

Fig. 2 - ECG fragments corresponding to periods of pre-crisis (a), first AFIB (b),

$\operatorname{AFL}(c)$, second AFIB (d), and post-crisis (e).

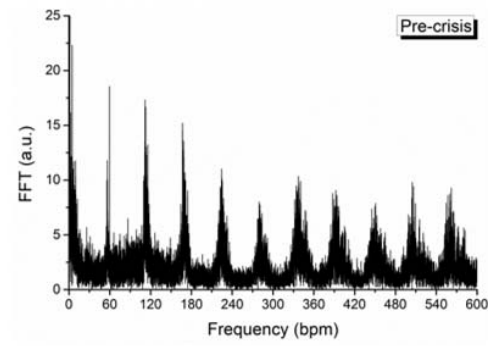

(a)

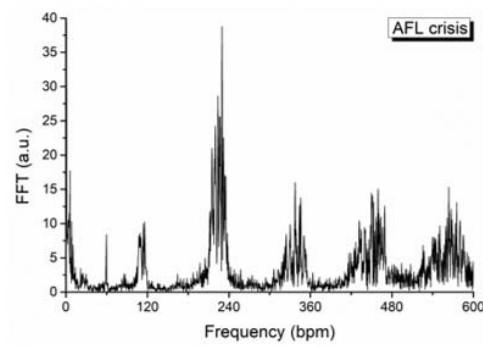

(c)

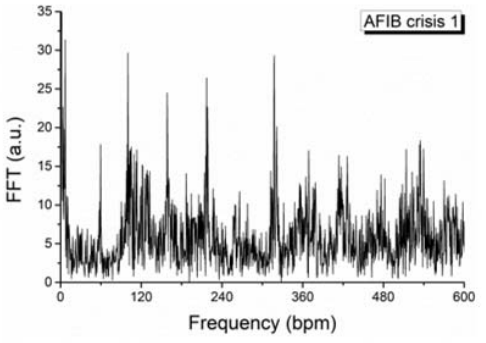

(b)

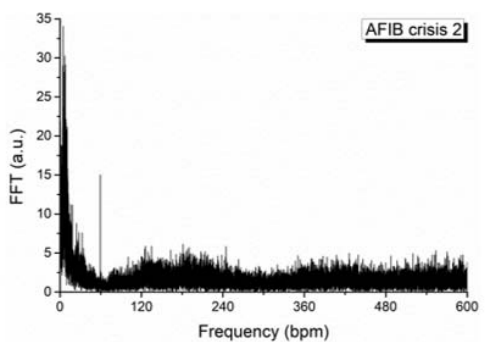

(d)

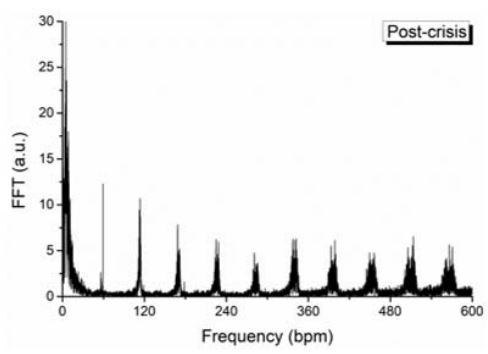

(e)

Fig. 3 - Fourier spectra for amplitudes of signals from Fig. 2. 
For the case shown in Fig. 2, we observe that in pre-crisis and postcrisis the pulse is normal. In AFIB1, we can see that the heart rate slowly increases, then it shows a much rapid increase in AFL, slowly decreasing afterwards in the AFIB2.

In the following, the results obtained by applying the mentioned nonlinear mathematical procedures are presented (Agop et al., 2004; Agop and Murgulet, 2007; Agop et al., 2008; Camm et al., 2009; Colodin et al., 2009; Iaizzo, 2015; Stoler, 1970; Stoler, 1971).

Fig. 3 shows the Fourier spectra for amplitudes of signals from Fig. 2.

The Fourier spectra shown above reveals to us the following: i) during pre-crisis (especially when compared to post-crisis), the signal shows a detectable noise, meaning that heart beats start to slowly become irregular, anticipating a crisis; ii) the graphs for AFIB crisis 1 and AFIB crisis 2 display the chaotic behavior of the heart (heart rate) during these crisis episodes. This can be explained through the fact that, during atrial flutter, the heart's upper chambers (atria) beat too quickly. This leads to a fast, but usually regular, heart rhythm. The specific Fourier spectrum confirms these findings. In post-crisis, the noise starts to decrease, thus showing a return to a relative normal and regular heartbeat.

Fig. 4 shows the systems dynamics attractors in the phase space reconstructed trough the delay time method, determined using the autocorrelation function.

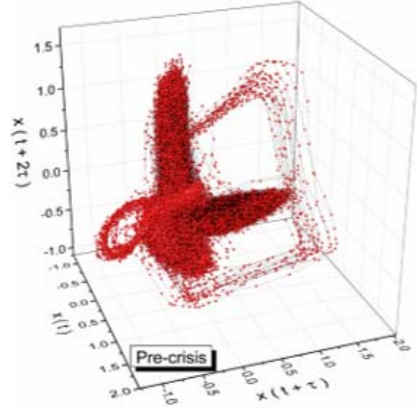

(a)

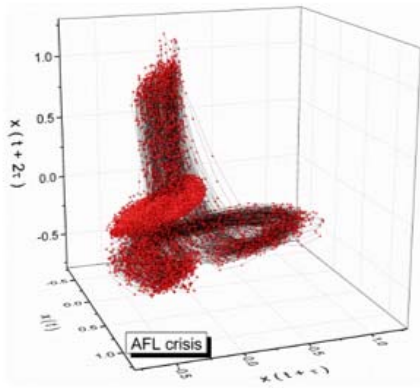

(c)

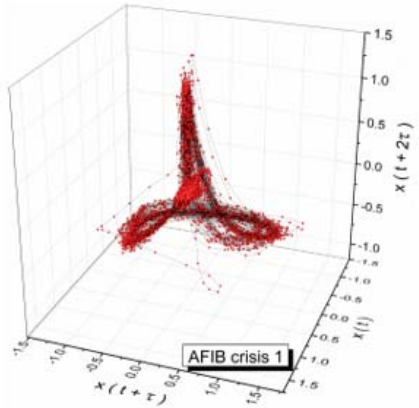

(b)

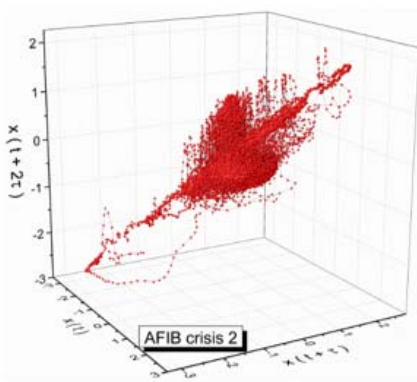

(d) 


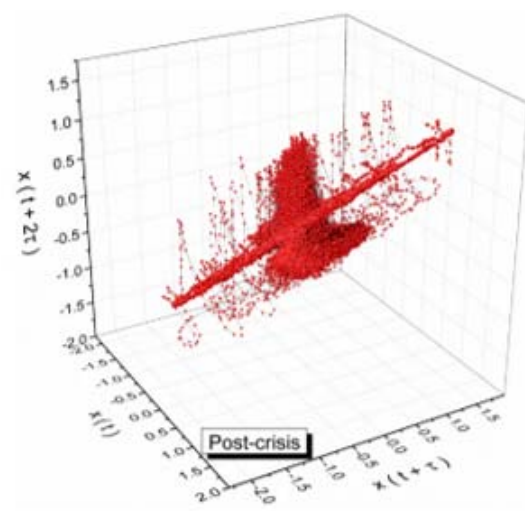

(e)

Fig. 4 - Systems dynamics attractors in the reconstructed phase space corresponding to signals from Fig. 2.

Let us observe the clear differences between the geometry (shape) of attractors from Figure 3, corresponding to the two atrial crises (AFIB 1 and 2), and also the notable differences between pre-crisis and post-crisis. Thus, we can guess that a defibrillator was used to restore normal heart rhythm.

\section{Conclusions}

Our results show that, by applying nonlinear dynamics mathematical procedures in the heart electrical activity analysis, we can obtain valuable information with respect to fibrillation crises and, possibly, other cardiac afflictions.

Therefore, we believe that our method can be used for developing new medical diagnosis models and also for tracking the evolution of heart diseases.

\section{REFERENCES}

Aflori M., Dimitriu D.G., Signal Analysis Using Nonlinear Dynamics Methods (in Romanian), in: Dumitrascu L., Dumitrascu I., Dorohoi D.O., Dimitriu D.G., Apreotesei G., Aflori M. (Eds.), Physics Complements for PhD Candidates (in Romanian), I, Iaşi, Tehnopress (2006).

Agop M., Ioannou P., Nica P., Radu C., Alexandru A., Vizureanu P., Fractal Characteristics of the Solidification Process, Materials Transactions, 45, 972975 (2004).

Agop M., Murgulet C., El Naschie’s Epsilon((Infinity)) Space-Time and Scale Relativity Theory in the Topological Dimension $D=4$, Chaos Solitons \& Fractals, 32, 1231-1240 (2007). 
Agop M., Paun V., Harabagiu A., El Naschie's Epsilon((Infinity)) Theory and Effect of Nanoparticle Clustering on the Heat Transport in Nanofluids, Chaos Solitons \& Fractals, 37, 1269-1278 (2008).

Arce G.R., Nonlinear Signal Processing: A Statistical Approach, Hoboken, Wiley (2004).

Badescu M., Ciocoiu M., Mocanu V., Practical Physiopathology Notions (in Romanian), Iaşi, Gr. T. Popa Publishing House (2016).

Camm A.J., Lüscher T.F., Serruys P.W., Eds., The ESC Textbook of Cardiovascular Medicine, 2nd Ed., Oxford, Oxford University Press (2009).

Christini D.J., Collins J.J., Hall K., Glass L., Controlling Cardiac Arrhythmias: The Relevance of Nonlinear Dynamics, in Moss F., Gielen S. (Eds.), Handbook of Biological Physics, 4, Amsterdam, Elsevier, 205-227 (2001).

Colotin M., Pompilian G.O., Nica P., Gurlui S., Paun V., Agop M., Fractal Transport Phenomena through the Scale Relativity Model, Acta Physica Polonica A, 116, 157-164 (2009).

Eckmann J.P., Kamphorst S.O., Ruelle D., Recurrence Plots of Dynamical Systems, Europhysics Letters, 4, 973-977 (1987).

Guevara M.R., Glass L., Shrier A., Phase Locking, Period-Doubling Bifurcations, and Irregular Dynamics in Periodically Stimulated Cardiac Cells, Science, 214, 1350-1353 (1981).

Haulica I., Human Physiology, Bucharest, Medical Publishing House (2007).

Iaizzo P.A. (Ed.) Handbook of Cardiac Anatomy, Physiology, and Devices, 3rd Ed., New York, Springer (2015).

Luther S., Fenton F.H., Kornreich B.G., Squires A., Bittihn P., Hornung D., Zabel M., Flanders J., Gladuli A., Campoy L., Cherry E.M., Luther G., Hasenfuss G., Krinsky V.I., Pumir A., Gilmour Jr. R.F., Bodenschatz E., Low-Energy Control of Electrical Turbulence in the Heart, Nature, 475, 235-239 (2011).

Mané R., On the Dimension of Compact Invariant Sets of Certain Nonlinear Maps, Lecture Notes in Mathematics, 898, 230-242 (1981).

Nayak S.K., Bit A., Dey A., Mohapatra B., Pal K., A Review of the Nonlinear Dynamical System Analysis of Electrocardiogram Signal, Journal of Healthcare Engineering 2018, 1-19 (2018).

Nayfeh A.H., Balachandran B., Applied Nonlinear Dynamics. Analytical, Computational, and Experimental Methods, Weinheim, Wiley-VCH (2004).

Noble D., A Modification of the Hodgkin-Huxley Equations Applicable to Purkinje Fibre Action and Pace-Maker Potentials, Journal of Physiology, 160, 317-352 (1960).

Packard N.H., Crutchfield J.P., Farmer J.D., Shaw R.S., Geometry from a Time Series, Physical Review Letters, 45, 712-716 (1980).

Perc M., Nonlinear Time Series Analysis of the Human Electrocardiogram, European Journal of Physics, 26, 757-768 (2005).

Ritzenberg A.L., Adam D.R., Cohen R.J., Period Multiplying-Evidence for Nonlinear Behaviour of the Canine Heart, Nature, 307, 159-161 (1984).

Ruelle D., Chaotic Evolution and Strange Attractors, Cambridge, Cambridge University Press (1989).

Stoler D., Equivalence Classes of Minimum Uncertainty Packets, Phys. Rev. D 1, 3217 3219 (1970). 
Stoler D., Generalized Coherence States, Phys. Rev. D 4, 2309 (1971).

Su Z.-Y., Wu T., Yang P.-H., Wang Y.-T., Dynamic Analysis of Heartbeat Rate Signals of Epileptics Using Multidimensional Phase Space Reconstruction Approach, Physica A, 387, 2293-2305 (2008).

Takens F., Detecting Strange Attractors in Turbulence, Lecture Notes in Mathematics, 898, 366-381 (1981).

Vagos M.R.S.S., van Herck I.G.M., Sundnes J., Arevalo H.J., Edwards A.G., Koivumäki J.T., Computational Modeling of Electrophysiology and Pharmacotherapy of Atrial Fibrillation: Recent Advances and Future Challenges, Frontiers in Physiology, 9, 1-29 (2018).

Young H., Benton D., We Should be Using Nonlinear Indices when Relating Heart-Rate Dynamics to Cognition and Mood, Scientific Reports, 5, 1-16 (2015).

https://physionet.org/physiobank/database/chbmit/.

\section{UN NOU MODEL TEORETIC PENTRU EVALUAREA FIBRILAȚIILOR ATRIALE}

\section{(Rezumat)}

În prezenta lucrare se dezvoltă o nouă metodă de evaluarea a fibrilațiilor atriale, pe baza unor proceduri matematice specifice dinamicii neliniare. Această metodă ar putea fi utilizată pentru dezvoltarea de noi modele pentru diagnosticul și monitorizarea bolilor cardiace, folosind tipare specifice. 